\title{
AJCC 8th edition prognostic staging provides no better discriminatory ability in prognosis than anatomical staging in triple negative breast cancer
}

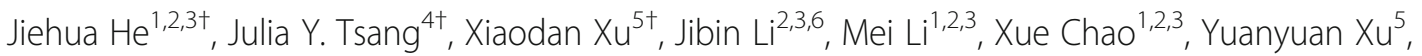
Rongzhen Luo ${ }^{1,2,3}$, Gary M. Tse ${ }^{4}$ and Peng Sun ${ }^{1,2,3^{*}}$ [D

\begin{abstract}
Background: We retrospectively compared the prognostic value between the AJCC 8th edition anatomic (AS) and prognostic staging (PS) system for triple negative breast cancer (TNBC) in a cohort from two involved institutions and a large population database.

Methods: Clinicopathological data of TNBCs were identified in two involved institutions (SYSUCC-PWH cohort). Data from SEER database during 2010-2015 was also accessed. We restaged all cases into AS and PS group according to the AJCC 8th staging system.

Results: A total of 611 and 31,941 TNBCs were identified in two cohorts, with a median follow-up of 53.5 and 27 months respectively. PS upstaged $46.1 \%$ of patients in SYSUCC-PWH cohort, and $62.4 \%$ in SEER cohort. No significant difference was observed in C index between AS and PS models for disease-specific survival (DSS), progression-free survival (PFS) or overall survival (OS) in either cohort. X2 statistic and Hazard Ratio for PFS, DSS and OS showed better discrimination between IA and IB, IIB and IIIA, IIIA and IIIB in AS model than PS model. Besides, patients with IIIC unchanged stage showed worse PFS compared to those with AS IIIA or IIIB upstaged to PS IIIC in both cohorts $(p=0.049, p<0.001)$.
\end{abstract}

Conclusions: Our findings demonstrated that prognostic staging system did not provide better discriminatory ability in predicting TNBCs prognosis than anatomic staging system.

Keywords: Triple negative breast cancer, AJCC 8th, Prognostic stage, Anatomic stage

\section{Background}

Cancer staging helps clinicians to determine prognosis and design treatment plans for individual patient. Since 1977, the American Joint Committee on Cancer (AJCC) staging system for breast cancer (BC) has assigned stage based on anatomical parameters, including tumor size

\footnotetext{
* Correspondence: sunpeng1@sysucc.org.cn

${ }^{\dagger}$ Jiehua He, Julia Y Tsang and Xiaodan Xu contributed equally to this paper as co-first authors.

${ }^{1}$ Department of Pathology, Sun Yat-sen University Cancer Center, Guangzhou, China

${ }^{2}$ State Key Laboratory of Oncology in South China, Guangzhou, China

Full list of author information is available at the end of the article
}

and extent of tumors $(\mathrm{T})$, Lymph node involvement $(\mathrm{N})$ and the presence or absence of distant metastasis (M). Once the T, N, and $\mathrm{M}$ are determined, they are combined for assigning the overall anatomic stage (AS) of 0 , I, II, III, IV. However, a significant limitation of AS for $\mathrm{BCs}$ is that it does not include the biologic factors and may not represent the $\mathrm{BC}$ biologic behavior sufficiently.

Identification of BCs molecular subtypes and significant progress in genomics studies have led to a better understanding of $\mathrm{BCs}$ biologic behavior, which provides valuable information for the individualized treatment of BCs. Therefore, AJCC 8th Edition Cancer Staging

(c) The Author(s). 2020 Open Access This article is distributed under the terms of the Creative Commons Attribution 4.0 International License (http://creativecommons.org/licenses/by/4.0/) which permits unrestricted use, distribution, and reproduction in any medium, provided you give appropriate credit to the original author(s) and the source, provide a link to the Creative Commons license, and indicate if changes were made. The Creative Commons Public Domain Dedication waiver (http://creativecommons.org/publicdomain/zero/1.0/) applies to the data made available in this article, unless otherwise stated. 
Manual has updated with a prognostic stage (PS) group for breast cancer by incorporating traditional TNM anatomic parameters and additional biologic factors, including estrogen receptor (ER) and progesterone receptor (PR) status, HER2 status, tumor grade and Recurrence Scores (Oncotype Dx), which are known to have predictive and prognostic value $[1,2]$. Previous validation studies have demonstrated that AJCC 8th edition PS provided more accurate prognostic information than AS in HR positive and HER2 positive breast cancer [3-6]. However, for triple negative breast cancer (TNBC) patients that lack expression of ER, PR and HER2, whether PS exhibits a better prognostic value than AS in these patients is still debatable $[7,8]$.

In this study, we retrospectively compared the prognostic value between the AJCC 8th edition AS and PS for TNBCs in a large local cohort (Sun Yat-sen University Cancer Center and Prince of Wales Hospital) and performed a SEER population-based analysis. We aim to examine whether this novel PS system provide more accurate risk stratification in overall survival, disease-specific survival, progressionfree survival than the AS system.

\section{Methods}

\section{Identification of SYSUCC-PWH cohort}

Clinicopathologic data, including tumor histologic grade, pathologic $\mathrm{T}$ and $\mathrm{N}$ categories, and ER, PR, and HER2 status, for patients who underwent surgery as the initial intervention with pathological confirmed invasive breast cancer in Sun Yat-sen University Cancer Center (SYSUCC) during 2005-2013 and Prince of Wales Hospital (PWH) during 2002-2008 were recorded. Patients receiving neoadjuvant therapy, and those with stage IV disease, re-operation for local recurrence,or incomplete or unknown clinicopathologic data were excluded.

All pathologic specimens were reviewed by experienced pathologists to confirm the clinicopathologic characteristics including the tumor size, axillary nodal status, resection margin. ER, PR, and HER2 status were determined according to immunohistochemical (IHC) staining. For patients before 2010, ER and PR status was reclassified as negative using a cut-off of $1 \%$ according to the American Society of Clinical Oncology/College of American Pathologists (ASCO/CAP) guidelines [9]. HER2 status was define as negative with $0,1+$ on IHC as well as $2+$ on IHC without HER2 gene amplification on fluorescence in situ hybridization (FISH) [10].

\section{Identification of SEER cohort}

The SEER 18 Registry Research Data was accessed through the SEER*stat (Version 8.3.5) to extract cases in this cohort [11]. Since the HER2 status was not available for cases before 2010, the cases from SEER database was limited to the duration during 2010-2015. "Breast" was selected in side recode ICD-O-3/WHO 2008 category and "Triple Negative" was selected in Breast Subtype $(2010+)$ category. Clinicopathologic data including age at diagnosis, sex, year of diagnosis, primary site, histologic type, histologic grade, AJCC 7th edition stage group (T, N, M), survival months, cause-specific death classification, treatment information, such as surgery, chemotherapy and radiotherapy, were collected. Cases with stage IV disease and those with incomplete or unknown clinicopathologic data were excluded.

\section{Statistical analysis}

PS and AS were determined for cases in both SYSUCCPWH cohort and SEER cohort according to the AJCC 8 th edition staging manual. Cases with histologic grade of grade III (poorly differentiated) and IV (Undifferentiated; anaplastic) in SEER cohort were both classified as G3 in PS system. Distributions of AS and PS were compared in each cohort. We evaluated the number of patients with alterative stage by the PS system. PFS was define as the duration between date of diagnosis to date of disease progression or last contact. DSS was define as the duration between date of diagnosis to date of death because of breast cancer progression or last contact. OS was define as the duration between date of diagnosis to date of death or last contact. PFS and DSS according to AS and PS were compared for SYSUCC-PWH cohort. OS and DSS between the stages were compared for SEER cohort.

The log-rank test was used to compare differences of survival rates between groups. The Harrell concordance index (C index) was calculated using SAS (Version 9.4) to measure the model's predictive performance for the AS and PS models. A higher C index indicates a better predictive performance. Significance between the $\mathrm{C}$ index of AS and PS models was determined using $t$ test. The $\mathrm{X}^{2}$ statistic of the log-rank test was used to further calculate the discrimination between groups. A larger $\chi^{2}$ statistic indicates further distance between survival curves, and its $P$ value reflects the statistical significance of this distance. The relationship of AS and PS with DSS, PFS or OS was modeled using a Cox proportional hazards regression model. The results were expressed in hazard ratios (95\% CIs). Two-tailed $p$ value $<0.05$ was considered statistically significant, and IBM SPSS (Version 21.0) was used for analysis.

\section{Results}

A total of 611 patients with stage I to IIIC TNBC were identified in the SYSUCC-PWH cohort, of which 427 
and 184 patients were from Sun Yat-sen University Cancer Center (SYSUCC) and Prince of Wales Hospital (PWH) respectively. All patients were assigned a PS. Compared to AS, PS upstaged 282 patients (46.1\%) and, unchanged stage was in 329 patients (53.9\%). No patient showed downstaged PS in this cohort. For those with upstaged PS, 232 patients $(232 / 282,82.3 \%)$ showed one stage up (including IA to IB, IIB to IIIA, IIIA to IIIB and IIIB to IIIC) and 50 patients (50/282, 17.7\%) showed two stages up (IIIA to IIIC).

A total of 31,941 patients with stage I to IIIC TNBC were identified in the SEER cohort. Compared to AS, PS upstaged 19,924 patients (62.4\%), and unchanged stage was in 12,009 patients (37.6\%). Eight patient showed downstaged PS (IIIC to IIIB) in this cohort. For those with upstaged PS, 17771 out of 19,924 patients (89.2\%) showed one stage up (including IA to IB, IIB to IIIA, IIIA to IIIB and IIIB to IIIC) and 2153 patients (10.8\%) showed two stages up (IIIA to IIIC). Clinicopathologic characteristics and staging alterations for patients in both cohorts are listed in Additional file 3: Tables S1 and S2.

Survival analyses were conducted in both cohorts and survival curves are shown in Fig. 1. The median followup was 53.5 months for the SYSUCC-PWH cohort and 27 months for the SEER cohort. Five-year DSS and PFS for SYSUCC-PWH cohort by AS and PS are summarized in Table 1. Five-year DSS and OS for SEER cohort by AS and PS are summarized in Table 2. In SYSUCCPWH cohort, the C index for AS was 0.83 (95\% CI $0.63-0.98)$ in DSS and 0.80 (95\% CI $0.59-0.96)$ in PFS. The C index for PS was 0.84 (95\% CI 0.63-0.98) in DSS and 0.82 (95\% CI 0.62-0.97) in PFS (Fig. 1). No significant difference of $\mathrm{C}$ index between AS and PS models, reflecting that PS model may not be a more predictive accurate model for either DSS $(p=0.943)$ or PFS ( $p=$ $0.887)$ than AS model. Similar outcomes were observed in SEER cohort, the C index for AS was 0.86 (95\% CI $0.81-0.89$ ) in DSS and 0.90 (95\% CI $0.87-0.92$ ) in OS. The C index for PS was 0.85 (95\% CI $0.81-0.89$ ) in DSS and 0.90 (95\% CI 0.87-0.92) in OS. No significant difference of $\mathrm{C}$ index between AS and PS models again in either DSS $(p=0.95)$ or OS $(p=0.98)$.

The $\chi^{2}$ statistic was applied to evaluate the discriminatory power between different stages among AS and PS in both cohorts. In the SYSUCC-PWH cohort, $\chi^{2}$ on PFS between IIA and IIB for AS was $10.36(p=0.0013)$ and that for PS was similar, showing a $\chi^{2}$ of $10.05(p=$ 0.0015). Comparing between IIB and IIIA, $X^{2}$ on PFS was $5.765(P=0.0163)$ for AS while that for PS was $0.6587(P=0.417)$. Similarly, between IIIA and IIIB, AS model also displayed a larger $\chi^{2}$ statistic than PS on DSS $\left(\chi^{2=} 4.204, P=0.0403\right.$ vs $\left.\chi^{2=} 1.239, P=0.2656\right) \cdot X^{2}$ statistic and $P$ value on pair-wise stage comparison of the cohort are shown in Table 3.

In the SEER cohort, compared to PS model, $x^{2}$ statistic on DSS of AS model showed a larger and statistically significant difference between IA and IB (24.94, $P<0.0001$ vs $1.272, P=0.2593)$, IIA and IIB (137.6, $P<0.0001$ vs $23.93, P<0.0001$ ), IIB and IIIA (98.42, $P<0.0001$ vs $0.249, P=0.6178)$ as well as IIIA and IIIB (97.38 $P<0.0001$ vs $9.91, P=0.0016)$. However, PS model displayed larger $X^{2}$ statistic on DSS than AS model between IB and IIA $(0.0045, P=0.9461$ vs 220 , $P<0.0001)$ as well as IIIB and IIIC $(0.68, P=0.4088$ vs 21.1, $P<0.0001)$. Similar results were also observed on the discrimination between stages for OS. $X^{2}$ statistic and $P$ value on pair-wise stage comparison of the cohort are shown in Table 4.

\section{A-D. SYSUCC-PWH Cohort}

A

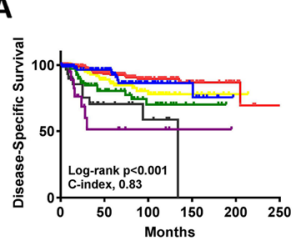

B
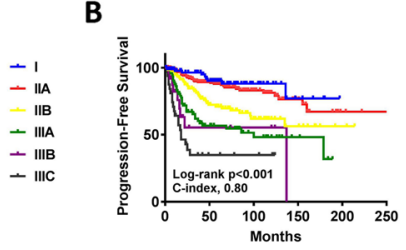

C

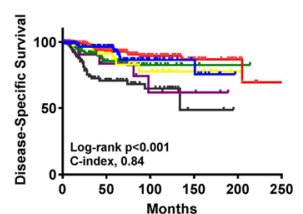

D

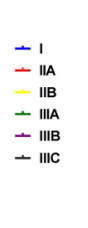

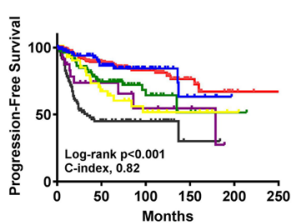

E-F. SEER Cohort

E
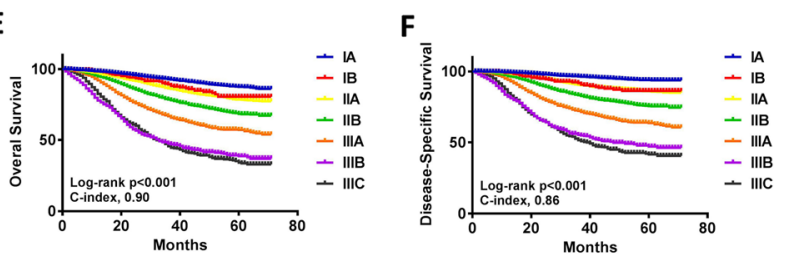

G

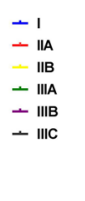

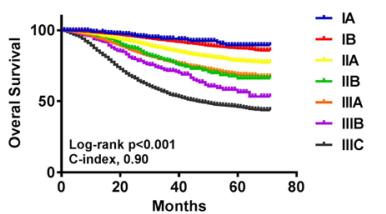

H

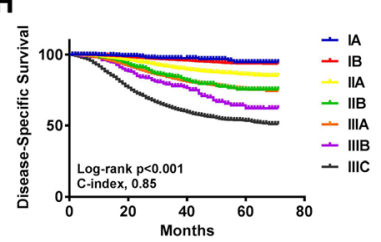

Fig. 1 Kaplan-Meier curves of DSS and PFS for the SYSUCC-PWH cohort in anatomical staging system (a-b) and prognostic staging system (c-d). Kaplan-Meier curves of OS and DSS for the SEER cohort in anatomical staging system (e-f) and prognostic staging system (g-h) 
Table 15 -year Progression-Free Survival and Disease-Specific Survival by Anatomic and Prognosis Stage of TNBCs included in SYSUCC-PWH cohort $(N=611)$

\begin{tabular}{|c|c|c|c|c|c|c|c|}
\hline & Stage & No. at risk & 5-year PFS (95\% Cl) & $p$-value & No. at risk & 5-year DSS (95\% Cl) & $p$-value \\
\hline \multirow[t]{8}{*}{$\overline{\mathrm{AS}}$} & $I\left(A^{*}\right)$ & 100 & $86.5(76.2,92.5)$ & $<0.001^{* *}$ & 100 & $92.9(83.4,97.0)$ & $0.001^{* *}$ \\
\hline & $\|$ & 371 & $82.0(77.0,86.0)$ & $0.001^{\#}$ & 371 & $90.6(86.3,93.6)$ & $0.080^{\#}$ \\
\hline & $\| \mathrm{A}$ & 240 & $87.9(82.4,91.9)$ & & 240 & $92.7(87.7,95.7)$ & \\
\hline & $\| B$ & 131 & $71.0(60.9,79.0)$ & & 131 & $86.2(76.8,92.0)$ & \\
\hline & III & 140 & $50.9(41.1,59.8)$ & $0.045^{\Delta}$ & 140 & $74.1(64.2,81.7)$ & $0.099^{\Delta}$ \\
\hline & $\| I I A$ & 78 & $56.6(43.7,67.6)$ & & 78 & $80.4(67.8,88.5)$ & \\
\hline & $\| \mathrm{IIB}$ & 29 & $55.2(28.6,75.4)$ & & 29 & $51.4(23.1,73.9)$ & \\
\hline & IIIC & 33 & $34.9(18.3,52.2)$ & & 33 & $70.4(46.9,85.0)$ & \\
\hline \multirow[t]{10}{*}{ PS } & I & 100 & $86.5(76.2,92.5)$ & $<0.001^{* *}$ & 100 & $92.9(83.4,97.0)$ & $<0.001^{* *}$ \\
\hline & $\mathrm{IA}$ & 3 & NA & NA & 3 & NA & NA \\
\hline & $\mathrm{IB}$ & 97 & $86.0(75.4,92.3)$ & & 97 & $92.7(88.5,97.0)$ & \\
\hline & $\|$ & 291 & $83.9(78.3,88.2)$ & $0.002^{\#}$ & 291 & $91.9(87.2,94.9)$ & $0.209^{\#}$ \\
\hline & $\| \mathrm{A}$ & 240 & 87.9(82.4,91.9) & & 240 & $92.7(89.7,95.7)$ & \\
\hline & $\| B$ & 51 & $64.1(46.1,77.5)$ & & 51 & $87.3(79.4,95.1)$ & \\
\hline & III & 220 & $60.1(52.3,66.9)$ & $<0.001^{\triangle}$ & 220 & $78.2(70.7,83.9)$ & $0.023^{\triangle}$ \\
\hline & $\| \mathrm{A}$ & 83 & $74.7(62.2,83.6)$ & & 83 & $85.5(78.7,92.3)$ & \\
\hline & $\| I B$ & 26 & $73.7(50.4,87.3)$ & & 26 & $83.5(72.5,94.5)$ & \\
\hline & $\| I I C$ & 111 & $44.9(34.0,55.1)$ & & 111 & $70.7(61.8,79.7)$ & \\
\hline
\end{tabular}

**Log-rank test comparing proportions among all stage; ${ }^{*}$ Log-rank test comparing proportions among Stage II; ${ }^{\triangle}$ Log-rank test comparing proportions among Stage III; *No case was classified as anatomic stage IB in this cohort

TNBC triple negative breast cancer, AS anatomic stage, PS prognosis stage, PFS progression-free survival, DSS disease specific survival, CI confidence interval

Furthermore, we also compare survival data between patients with unchanged stage and patients with upstaged PS. In SYSUCC-PWH cohort, patients with IIIC unchanged stage showed worse survival outcome than those patients with AS IIIA or IIIB upstaged to PS IIIC in PFS $(p=0.049$, Fig. $2 b)$, but not in DSS $(p=0.515$, Fig. 2a). Similarly, in SEER cohort, patients with IIIC unchanged stage showed worse survival outcome in both OS and DSS when compared to patients with AS IIIA or IIIB upstaged to PS IIIC $(p<0.001$, Fig. 2e-f). Besides, patients with IIA unchanged stage also showed worse OS than patients with AS IA or IB upstaged to PS IIA ( $p=0.0083$, Fig. 2 c). On the other hand, we found no significant difference in either DSS, PFS or OS between patients with IIB unchanged stage and those with AS IIB upstaged to PS IIIA in both cohorts. Survival curve and $p$ value are shown in Additional file 1: Figure S1 and Additional file 2: Figure S2.

A Cox proportional hazards regression model was then used to look into the Hazard Ratio (HR) for DSS, PFS or OS by stage in both cohorts. The hazard ratios for anatomic and prognostic stages in both cohorts are summarized in Additional file 3: Tables S2, S3 and S4.

\section{Discussion}

The greatest change of AJCC 8th edition cancer staging system is the incorporation of tumor grade, and ER, PR, and HER2 status, as well as genomic assays, which are generally accepted as important predictive and prognostic factors in clinical practice [9-14]. This mean that prognostic stage is more likely to reflect individual prognosis when patients receive the recommended treatment strategies $[1,2,6]$.

Previous studies have demonstrated that the PS model provided a better prognosis value than the AS model in individuals with breast cancer $[3,4,6,15]$, including the subgroup analysis for ER positive [5] and HER2 positive BCs [16]. This may due to the selection of genomic low risk patients and the improved effectiveness of endocrine therapy and anti-HER2 therapy. When looking into patients with TNBC, who predominantly receive chemotherapy, are more likely to have mid-high nuclear grade and worse prognosis for overall survival and disease-free survival $[17,18]$. It is not surprising that, 46.1 and $62.4 \%$ TNBC patients were upstaged from AS to PS model in our two study cohorts. The upstaged rate in TNBC is greater than that reported for all breast cancer (6.8$34.7 \%$ approximately) $[3,4,6,15]$. On the other hand, only 8 out of 31,941 patients with TNBC in SEER cohort were downstaged from AS IIIC to PS IIIB because of the low nuclear grade (grade 1). Compared to the reported downstaged rate of $23.4-35.6 \%$ approximately in overall breast cancer $[3,4,6,15]$, 
Table 2 5-year Overall Survival and Disease-Specific Survival by Anatomic and Prognosis Stage of TNBCs included in SEER cohort $(N=31,941)$

\begin{tabular}{|c|c|c|c|c|c|c|c|}
\hline & Stage & No. at risk & 5-year OS (95\% Cl) & $p$-value & No. at risk & 5-year DSS (95\% CI) & $p$-value \\
\hline \multirow[t]{10}{*}{ AS } & I & 12,700 & $87.2(86.2,88.0)$ & $<0.001^{* *}$ & 12,700 & $93.5(82.8,94.2)$ & $<0.001^{* *}$ \\
\hline & IA & 12,293 & $87.4(86.5,88.3)$ & $0.004^{*}$ & 12,293 & $93.8(93.1,94.4)$ & $0.001^{*}$ \\
\hline & IB & 407 & $80.4(73.9,85.5)$ & & 407 & $86.2(80.6,90.2)$ & \\
\hline & $\|$ & 14,292 & $75.3(74.2,76.4)$ & $<0.001^{\#}$ & 14,292 & $82.7(81.8,83.7)$ & $<0.001^{\#}$ \\
\hline & $\| \mathrm{A}$ & 9713 & $78.5(77.2,79.7)$ & & 9713 & 86.0(84.9,87.0) & \\
\hline & $\| \mathrm{B}$ & 4579 & $68.3(66.1,70.4)$ & & 4579 & 75.5(73.5,77.4) & \\
\hline & III & 4949 & $46.8(44.8,48.7)$ & $<0.001^{\Delta}$ & 4949 & $54.0(52.0,56.0)$ & $<0.001^{\Delta}$ \\
\hline & IIIA & 2515 & $57.1(54.3,59.7)$ & & 2515 & $63.6(60.8,66.2)$ & \\
\hline & IIB & 1164 & $38.5(34.5,42.5)$ & & 1164 & $47.0(42.6,51.2)$ & \\
\hline & $\mathrm{IIIC}$ & 1270 & $34.3(30.5,38.2)$ & & 1270 & $41.4(37.3,45.2)$ & \\
\hline \multirow[t]{10}{*}{ PS } & I & 12,293 & 87.4(86.5,88.3) & $<0.001^{* *}$ & 12,293 & 93.8(93.1,94.4) & $<0.001^{* *}$ \\
\hline & IA & 407 & $89.4(83.6,93.2)$ & $0.212^{*}$ & 407 & $94.6(89.2,97.3)$ & $0.259^{*}$ \\
\hline & $\mathrm{IB}$ & 11,886 & 87.4(86.5,88.3) & & 11,886 & 93.8(93.1,94.4) & \\
\hline & $\|$ & 10,726 & $77.9(76.7,79.1)$ & $<0.001^{\#}$ & 10,726 & $85.5(84.4,86.5)$ & $<0.001^{\#}$ \\
\hline & $\| \mathrm{A}$ & 10,120 & 78.6(77.3,79.8) & & 10,120 & $86.0(85.0,87.0)$ & \\
\hline & $\| B$ & 606 & $67.4(61.2,72.9)$ & & 606 & $75.4(69.3,80.4)$ & \\
\hline & III & 8922 & $56.3(54.8,57.8)$ & $<0.001^{\Delta}$ & 8922 & $63.5(62.0,65.0)$ & $<0.001^{\Delta}$ \\
\hline & IIA & 3986 & $68.7(66.4,70.8)$ & & 3986 & $75.6(73.4,77.6)$ & \\
\hline & $\| \mathrm{IIB}$ & 365 & $56.3(47.8,64.0)$ & & 365 & $62.1(53.0,70.0)$ & \\
\hline & IIIC & 4571 & $45.9(43.9,48.0)$ & & 4571 & $53.3(51.2,55.4)$ & \\
\hline
\end{tabular}

**Log-rank test comparing proportions among all stage; Log-rank test comparing proportions among Stage l; " Log-rank test comparing proportions among Stage II; ${ }^{\triangle}$ Log-rank test comparing proportions among Stage III

TNBC triple negative breast cancer, AS anatomic stage, PS prognosis stage, OS overall survival, DSS disease specific survival, CI confidence interval

rarely patients with TNBC were downstaged in PS model.

Weiss, et al. [6] found that $13.6 \% \mathrm{BC}$ patients could not be assigned to a prognostic stage due to the presence of N1mi disease in patientsnwith tumors larger than $\mathrm{T} 1$ or uncategorized combinations of $\mathrm{T}$ and $\mathrm{N}$ categories with grade and HR and HER2 status. Some subsequent changes had been made and demonstraded in the AJCC 8th Edition Updates and Corrections, that N1mi disease in patients with T2, T3 and T4 cancers includes N1mi. In the present study, all TNBCs were perfectly assigned to a proper prognostic stage according to the criterion. However, the results of present study in both TNBC cohorts do not show better prognostic value for PS model compared to the traditional AS model. PS model showed better discrimination for both OS and

Table 3 The $x 2$ statistic on Disease-Specific Survival and Progression-Free Survival for Anatomic and Prognostic Stage of TNBCs included in SYSUCC-PWH cohort $(N=611)$

\begin{tabular}{|c|c|c|c|c|c|c|c|c|}
\hline \multirow[t]{3}{*}{ Stage } & \multicolumn{4}{|c|}{ Disease-Specific Survival } & \multicolumn{4}{|c|}{ Progression-Free Survival } \\
\hline & \multicolumn{2}{|l|}{ AS } & \multicolumn{2}{|l|}{ PS } & \multicolumn{2}{|l|}{$\overline{A S}$} & \multicolumn{2}{|l|}{ PS } \\
\hline & $x^{2}$ & $p$-value & $x^{2}$ & $p$-value & $x^{2}$ & $p$-value & $x^{2}$ & $p$-value \\
\hline$I^{a}$ vs $\| A$ & 0.5876 & 0.4433 & 0.5876 & 0.4433 & 0.006753 & 0.9345 & 0.0068 & 0.9345 \\
\hline$\| A$ vs $\| B$ & 3.214 & 0.073 & 1.572 & 0.2099 & 10.36 & 0.0013 & 10.05 & 0.0015 \\
\hline$\| B$ vs IIA & 2.16 & 0.1417 & 0.0016 & 0.9681 & 5.765 & 0.0163 & 0.6587 & 0.417 \\
\hline$\| A$ vs $\| B$ & 4.204 & 0.0403 & 1.239 & 0.2656 & 0.5332 & 0.4653 & 0.7811 & 0.3768 \\
\hline$\| I B$ vs $\| I C$ & 0.2007 & 0.6541 & 0.7913 & 0.3737 & 1.922 & 0.1656 & 2.294 & 0.1299 \\
\hline
\end{tabular}

${ }^{a}$ No case was classified as anatomic stage IB in this cohort 
Table 4 The $\times 2$ statistic on Disease-Specific Survival and Overall Survival for Anatomic and Prognostic Stage of TNBCs included in SEER cohort $(N=31,941)$

\begin{tabular}{|c|c|c|c|c|c|c|c|c|}
\hline \multirow[t]{3}{*}{ Stage } & \multicolumn{4}{|c|}{ Disease-Specific Survival } & \multicolumn{4}{|c|}{ Overall Survival } \\
\hline & \multicolumn{2}{|l|}{$\overline{\mathrm{AS}}$} & \multicolumn{2}{|l|}{ PS } & \multicolumn{2}{|l|}{ AS } & \multicolumn{2}{|l|}{ PS } \\
\hline & $x^{2}$ & $p$-value & $x^{2}$ & $p$-value & $x^{2}$ & $p$-value & $x^{2}$ & $p$-value \\
\hline IA vs IB & 24.94 & $<0.0001$ & 1.272 & 0.2593 & 8.254 & 0.0041 & 1.561 & 0.2115 \\
\hline IB vs IIA & 0.0045 & 0.9461 & 220 & $<0.0001$ & 1.247 & 0.2641 & 183.3 & $<0.0001$ \\
\hline$\| A$ vs\|B & 137.6 & $<0.0001$ & 23.93 & $<0.0001$ & 109.5 & $<0.0001$ & 22.64 & $<0.0001$ \\
\hline$\| B$ vs $\| A$ & 98.42 & $<0.0001$ & 0.249 & 0.6178 & 92.07 & $<0.0001$ & 0.005236 & 0.9423 \\
\hline$\| \mathrm{A}$ vs $\| \mathrm{B}$ & 97.38 & $<0.0001$ & 9.91 & 0.0016 & 128.1 & $<0.0001$ & 11 & 0.0009 \\
\hline IIIB vs IIIC & 0.6823 & 0.4088 & 21.1 & $<0.0001$ & 0.0601 & 0.8063 & 21.44 & $<0.0001$ \\
\hline
\end{tabular}

TNBC triple negative breast cancer, $A S$ anatomic stage, $P S$ prognosis stage

DSS between IB and IIA as well as IIIB and IIIC. However, worse discrimination between IA and IB as well as IIB and IIIA were found in PS compared to AS model. Those upstaged from AS (IIIA/IIIB to IIIIC) showed a significant worse survival than those with unchanged PS (IIIC). Similarly, those upstaged to a higher PS (e.g. IIB to IIIA) did not show significant worse survival than PS stage equivalent to their original AS (IIB unchanged). Our findings demonstrated that the new prognostic staging system do not provide better discriminatory ability in predicting TNBCs prognosis than anatomic staging system.

Upstaged TNBCs by PS in the present study are mostly from AS IA, IIB, IIIA and IIIB, however, we noticed that those upstaged cases do not demonstrate the relevant worse survival outcomes. These findings are consistent with previous study by Liu, et al. [8]. On the contrary, contradictory studies by Li, et al. [7] and Luo, et al. [19] indicated that the PS system displayed a more optimistic prognostic stratification and predictability than traditional AS system. However, Li, et al. [7] applied a earlier version of AJCC 8th criterion without subsequent corrections in a small sample cohort including stage IV disease. They also excluded special types of invasive breast cancer, and no relevant statistical methods had been applied to further assess and compare prognostic ability of the two staging systems. Luo, et al. [19] used the goodness-of-fit test, included statistics as -2likelihood, AIC, and BIC, to describe the prediction capability of the two competing staging systems in TNBCs and found that new version of AJCC staging system were higher than before. However, they also mentioned that statistics such as AIC and BIC are not convertible to a clinical meaningful relevance. The calculation of the $\mathrm{C}$-index at different time points did not show significant differences in the two competing stage systems, which is in line with our observations.

The mainstay treatment of TNBC remains to be chemotherapy. Despite the upstages in PS, the clinical treatment decision will unlikely be changed for the time being $[6,20]$. So it is unnecessary to worry about the overtreatments for TNBCs. However, PS could not accurately stratify risk in TNBC, thus further updates should be required. Additional morphological and genomic information with prognostic significance in TNBCs have been demonstrated. Upcoming studies should consider the incorporation of biologic factors that closely related to the development of novel clinical therapies in TNBCs, for example, Poly-ADP-ribose polymerase inhibitors (PARPi) including Olaparib or Talazoparib and platinum therapy may provided a significant benefit over standard chemotherapy with respect to progressionfree survival for metastatic TNBCs with germline BRCA1/2 mutation [21-23]; Presence and increasing percentage of stromal tumor infiltrating lymphocytes (sTILs) $[24,25]$ are associated with better response to anthracycline-based neoadjuvant chemotherapy and improved long-term survival in TNBCs; Atezolizumab plus nab-paclitaxel prolonged progression-free survival among patients with metastatic triple-negative breast cancer in PD-L1-positive subgroup [26]. Moreover, activation PI3K/AKT signaling [27], AR expression [28], histopathological and molecular subtypes [29, 30] should also be considered for the modification of the prognostic staging system in TNBCs. One limitation of the present study is that we cannot acquire data regarding administration of PARPi and immunotherapy. As a retrospective study, we enrolled TNBCs diagnosed at the time during 2005-2015 (SYSUCC, 2005-2013; PWH, 2002-2008; SEER, 2010-2015), when PARPi and immunotherapy were not widely approved for clinical practice and the predominant treatment of TNBC remains to be chemotherapy and radiotherapy, especially in Asian countries. However, subsequent clinical trials, including EMBRACA [21] Trial, OlympiAD [22] Trial, PrECOG 0105 Trial [22] and IMpassion130 Trial [26], suggested the significant effects of novel treatment modalities especially for advanced TNBCs. Another limitation of the present study is 


\section{A-B SYSUCC-PWH Cohort}

A

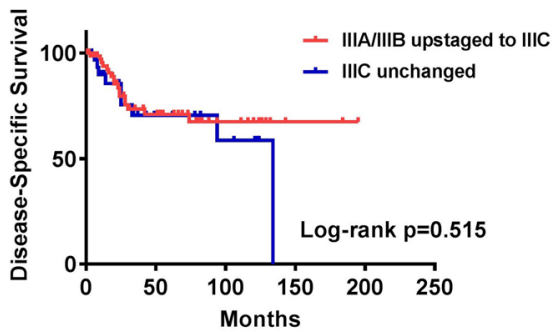

\section{C-F SEER Cohort}

C

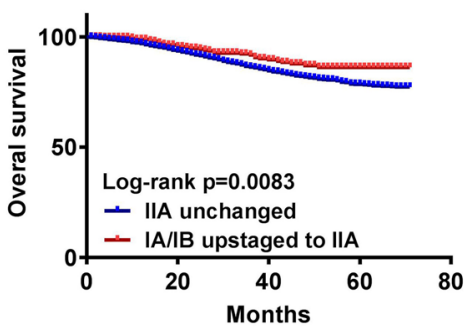

E

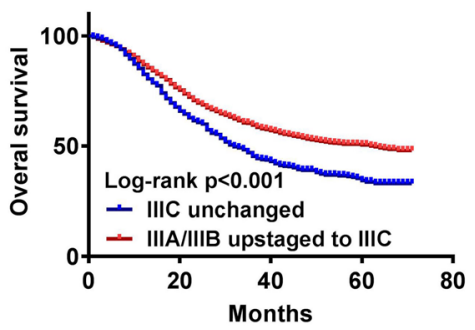

B

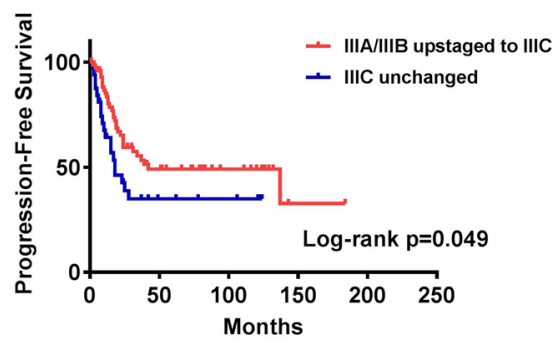

D

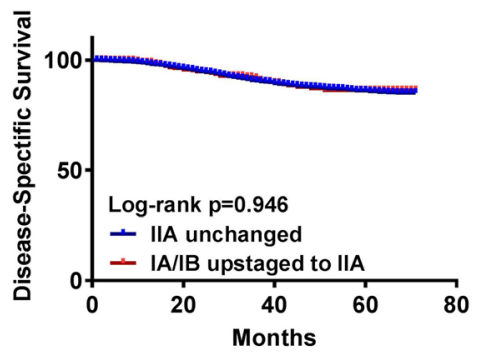

$\mathbf{F}$

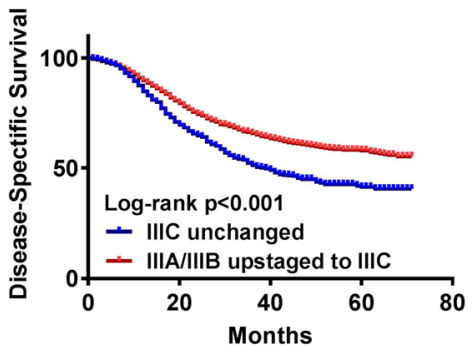

Fig. 2 Kaplan-Meier curves of DSS (a) and PFS (b) for the SYSUCC-PWH cohort between patients with IIIC unchanged and those with anatomical stage IIIA/IIIB upstaged to prognostic stage IIIC. Kaplan-Meier curves of OS (c) and DSS (d) for the SEER cohort between patients with IIA unchanged and those with anatomical stage IA/IB upstaged to prognostic stage IIA; Kaplan-Meier curves of OS (e) and DSS (f) for the SEER cohort between patients with IIIC unchanged and those with anatomical stage IIIA/IIIB upstaged to prognostic stage IIIC

that no case was classified as anatomic stage IB in SYSUCC-PWH cohort. In SEER cohort, we found that few cases of TNBC (1.3\%) were classified as AS IB (T0NmiM0 and T1N1miM0). So it can be happened that no AS IB case was found in the SYSUCC-PWH cohort with a smaller sample size. This may also due to the reluctance for pathologists to make the diagnosis of Nmi at the time of initial diagnosis. However, all the AS IB cases, regardless of the variety in histologic grade, are classified as the unchanged PS IB following the AJCC 8th criteria, which may not affect outcomes of the comparative study.

\section{Conclusions}

Our findings demonstrated that prognostic staging system did not provide better discriminatory ability in predicting TNBCs prognosis than anatomic staging system. The prognosis staging system should be further modified following the development of novel clinical therapies in TNBCs. 


\section{Supplementary information}

Supplementary information accompanies this paper at https://doi.org/10. 1186/s12885-019-6494-3

Additional file 1: Figure S1. Kaplan-Meier curves of DSS (A) and PFS (B) for the SYSUCC-PWH cohort between patients with IIB unchanged and those with anatomical stage IIB upstaged to prognostic stage IIIA.

Additional file 2: Figure S2. Kaplan-Meier curves of OS (A) and DSS (B) for the SEER cohort between patients with IA unchanged and those with anatomical stage IA upstaged to prognostic stage IB; Kaplan-Meier curves of OS (C) and DSS (D) for the SEER cohort between patients with IIB unchanged and those with anatomical stage IIB upstaged to prognostic stage IIIA.

Additional file 3: Table S1. Clinicopathologic characteristics of TNBCS included in SYSUCC-PWH cohort $(N=611)$ and SEER cohort $(N=31,941)$. Table S2. Alteration from Anatomic to Prognostic Stage of TNBCs included in SYSUCC-PWH cohort $(N=611)$ and SEER cohort $(N=31,941)$.

Table S3. Hazard Ratio for Disease-Specific Survival and Progression-Free Survival by Stage of TNBCS included in the SYSUCC-PWH cohort ( $N=$

611). Table S4. Hazard Ratio for Disease-Specific Survival and Overall Survival by Stage of TNBCs included in the SEER cohort $(N=31,941)$.

\section{Abbreviations}

AJCC: The American Joint Committee on Cancer; AS: Anatomic stage; BC: Breast cancer; DSS: Disease-specific survival; OS: Overall survival; PFS: Progression-free survival; PS: Prognostic stage; PWH: Prince of Wales Hospital; SEER database: Surveillance, epidemiology, and end results database; SYSUCC: Sun Yat-sen University Cancer Center; TNBC: Triple negative breast cancer

\section{Acknowledgements}

Not applicable.

\section{Authors' contributions}

Conceptualisation: PS, JH, JYT, GMT; Data acquisition: XX, ML, XC, YX, RL Methodology: PS, JH, JYT, JL,XX, GMT; Data analysis: PS, JYT, XX, JL,ML; Writing original draft and editing: JH, PS, JYT, XX, GMT, JH, JL; Data curation: PS, JYT, XX, GMT, JH; Project administration: PS, GMT, JH; Funding acquisition: PS. All authors read and approved the final manuscript.

\section{Funding}

This study was funded by the Guangdong Medical Research Foundation (A2018241) and National Natural Science Foundation of China (81902679) in the design of the study and collection, analysis, and interpretation of data and in writing the manuscript.

\section{Availability of data and materials}

The SEER-database is publicly available. The datasets generated and/or analysed during the current study are also available from the corresponding author on reasonable request.

\section{Ethics approval and consent to participate}

All procedures performed in studies involving human participants were in accordance with the ethical standards of the Sun Yat-sen University Cancer Center (SYSUCC) research committee and with the 1964 Helsinki declaration and its later amendments or comparable ethical standards. The formal written informed consent was obtained from all individual participants included in the study.

\section{Consent for publication}

Not applicable.

\section{Competing interests}

The authors declare that they have no competing interests.

\section{Author details}

'Department of Pathology, Sun Yat-sen University Cancer Center, Guangzhou, China. ${ }^{2}$ State Key Laboratory of Oncology in South China, Guangzhou, China. ${ }^{3}$ Collaborative Innovation Center for Cancer Medicine,
Guangzhou, China. ${ }^{4}$ Department of Anatomical and Cellular Pathology, Prince of Wales Hospital, The Chinese University of Hong Kong, Hong Kong, China. ${ }^{5}$ Department of Physiology, Zhongshan School of Medicine, Sun Yat-sen University, Guangzhou, China. ${ }^{6}$ Department of Clinical Research, Sun Yat-sen University Cancer Center, Guangzhou, China.

Received: 9 October 2019 Accepted: 23 December 2019

Published online: 06 January 2020

\section{References}

1. Hortobagyi GN, Connolly JL, Edge SB, et al. Breast. In: Amin MB, Edge S, Greene F, et al., editors. AJCC Cancer staging manual. 8th ed. New York: Springer International Publishing; 2016.

2. Hortobagyi GN, Edge SB, Giuliano A. New and important changes in the TNM staging system for breast Cancer. Am Soc Clin Oncol Educ Book. 2018; 38:457-67.

3. Abdel-Rahman O. Validation of the 8th AJCC prognostic staging system for breast cancer in a population-based setting. Breast Cancer Res Treat. 2018; 168(1):269-75.

4. Shao N, Xie C, Shi Y, Ye R, Long J, et al. Comparison of the 7th and 8th edition of American joint committee on Cancer (AJCC) staging systems for breast cancer patients: a surveillance, epidemiology and end results (SEER) analysis. Cancer Manag Res. 2019;11:1433-42.

5. Wang M, Wu K, Zhang P, Zhang M, Ding A, et al. The prognostic significance of the Oncotype DX recurrence score in T1-2N1M0 estrogen receptor-positive HER2-negative breast Cancer based on the prognostic stage in the updated AJCC 8th edition. Ann Surg Oncol. 2019;26(5):1227-35.

6. Weiss A, Chavez-MacGregor M, Lichtensztajn DY, Yi M, Tadros A, et al. Validation study of the American joint committee on Cancer eighth edition prognostic stage compared with the anatomic stage in breast Cancer. JAMA Oncol. 2018:4(2):203-9.

7. Li JP, Zhang XM, Zhang YS, Zheng LH, Liu YJ. The prognostic value of the 8th edition of the American Joint Committee on Cancer (AJCC) staging system in triple-negative breast cancer. Neoplasma. 2019;66(5):810-817.

8. Liu YY, Yu TJ, Liu GY. The predictive value of the prognostic staging system in the 8th edition of the American joint committee on Cancer for triplenegative breast cancer: a SEER population-based analysis. Future Oncol. 2019;15(4):391-400.

9. Hammond ME, Hayes DF, Dowsett M, Allred DC, Hagerty KL, et al. American Society of Clinical Oncology/college of American pathologists guideline recommendations for immunohistochemical testing of estrogen and progesterone receptors in breast cancer. J Clin Oncol. 2010;28(16):2784-95.

10. Wolff AC, Hammond MEH, Allison KH, Harvey BE, Mangu PB, et al. Human epidermal growth factor receptor 2 testing in breast Cancer: American Society of Clinical Oncology/College of American Pathologists Clinical Practice Guideline Focused Update. J Clin Oncol. 2018:36(20):2105-22.

11. Surveillance, epidemiology and end results program. about the SEER program. http://seer.cancer.gov/about. Accessed 10 Oct 2016.

12. National Comprehensive Cancer Network. Clinical Practice Guidelines in Oncology: breast. Clinical Practice Guidelines in Oncology: breast. https:// www.nccn.org/professionals/physician_gls/pdf/breast.pdf. Published 2019. Accessed 5 Apr 2019.

13. Curigliano G, Burstein HJ, Winer EP, Gnant M, Dubsky P, et al. De-escalating and escalating treatments for early-stage breast cancer: the St. Gallen International Expert Consensus Conference on the Primary Therapy of Early Breast Cancer 2017. Ann Oncol. 2018;29(10):2153.

14. Krop I, Ismaila N, Andre F, Bast RC, Barlow W, et al. Use of biomarkers to guide decisions on adjuvant systemic therapy for women with early-stage invasive breast Cancer: American Society of Clinical Oncology clinical practice guideline focused update. J Clin Oncol. 2017;35(24):2838-47.

15. Wang M, Chen H, Wu K, Ding A, Zhang M, et al. Evaluation of the prognostic stage in the 8th edition of the American joint committee on Cancer in locally advanced breast cancer: an analysis based on SEER 18 database. Breast. 2018;37:56-63.

16. Zhou B, Xu L, Ye J, Xin L, Duan X, et al. The prognostic value of the 8th edition of the American joint committee on Cancer (AJCC) staging system in HER2-enriched subtype breast Cancer, a retrospective analysis. Anticancer Res. 2017:37(8):4615-21.

17. Dent R, Trudeau M, Pritchard Kl, Hanna WM, Kahn HK, et al. Triple-negative breast cancer: clinical features and patterns of recurrence. Clin Cancer Res. 2007;13:4429-34. 
18. Sharma S, Barry M, Gallagher DJ, Kell M, Sacchini V. An overview of triple negative breast cancer for surgical oncologists. Surg Oncol. 2015; 24(3):276-83.

19. Luo S, Wu Q, Chen H, Wang X, Chen Q, et al. Validation of the Prognostic Significance of the Prognostic Stage Group According to the Eighth Edition of American Cancer Joint Committee on Cancer Staging System in TripleNegative Breast Cancer: An Analysis From Surveillance, Epidemiology, and End Results 18 Database. J Surg Res. 2019;pii: S0022-4804(19):30741-3.

20. Denkert C, Liedtke C, Tutt A, von Minckwitz G. Molecular alterations in triple-negative breast cancer-the road to new treatment strategies. Lancet. 2017:389(10087):2430-42.

21. Litton JK, Rugo HS, Ettl J, Hurvitz SA, Gonçalves A, et al. Talazoparib in patients with advanced breast Cancer and a Germline BRCA mutation. N Engl J Med. 2018;379(8):753-63.

22. Robson M, Im SA, Senkus E, Xu B, Domchek SM, et al. Olaparib for metastatic breast Cancer in patients with a Germline BRCA mutation. N Engl J Med. 2017;377(6):523-33.

23. Telli ML, Jensen KC, Vinayak S, Kurian AW, Lipson JA, et al. Phase II study of gemcitabine, carboplatin, and iniparib as neoadjuvant therapy for triplenegative and BRCA1/2 mutation-associated breast cancer with assessment of a tumor-based measure of genomic instability: PrECOG 0105. J Clin Oncol. 2015:33(17):1895-901.

24. Loi S, Michiels S, Salgado R, Sirtaine N, Jose V, et al. Tumor infiltrating lymphocytes are prognostic in triple negative breast cancer and predictive for trastuzumab benefit in early breast cancer: results from the FinHER trial. Ann Oncol. 2014:25(8):1544-50

25. Adams S, Gray RJ, Demaria S, Goldstein L, Perez EA, et al. Prognostic value of tumor-infiltrating lymphocytes in triple-negative breast cancers from two phase III randomized adjuvant breast cancer trials: ECOG 2197 and ECOG 1199. J Clin Oncol. 2014;32(27):2959-66.

26. Schmid P, Adams S, Rugo HS, Schneeweiss A, Barrios CH, et al. Atezolizumab and nab-paclitaxel in advanced triple-negative breast Cancer. N Engl J Med. 2018;379(22):2108-21.

27. Hashimoto K, Tsuda H, Koizumi F, Shimizu C, Yonemori K, et al. Activated PI3K AKT and MAPK pathways are potential good prognostic markers in node-positive, triple-negative breast cancer. Ann Oncol. 2014;25(10):1973-9.

28. Ogawa Y, Hai E, Matsumoto K, Ikeda K, Tokunaga S, et al. Androgen receptor expression in breast cancer: relationship with clinicopathological factors and biomarkers. Int J Clin Oncol. 2008;13:431-5.

29. Jiang YZ, Ma D, Suo C, Shi J, Xue M, et al. Genomic and Transcriptomic landscape of triple-negative breast cancers: subtypes and treatment strategies. Cancer Cell. 2019;35(3):428-40.

30. Burstein MD, Tsimelzon A, Poage GM, Covington KR, Contreras A, et al. Comprehensive genomic analysis identifies novel subtypes and targets of triple-negative breast cancer. Cancer Cell. 2019;35(3):428-40.

\section{Publisher's Note}

Springer Nature remains neutral with regard to jurisdictional claims in published maps and institutional affiliations.

Ready to submit your research? Choose BMC and benefit from:

- fast, convenient online submission

- thorough peer review by experienced researchers in your field

- rapid publication on acceptance

- support for research data, including large and complex data types

- gold Open Access which fosters wider collaboration and increased citations

- maximum visibility for your research: over $100 \mathrm{M}$ website views per year

At $\mathrm{BMC}$, research is always in progress.

Learn more biomedcentral.com/submissions 